\title{
Overvaluing autonomous decision-making
}

\author{
Peter Lepping and Bevinahalli Nanjegowda Raveesh
}

\section{Summary}

Current capacity-based legislation and practice overvalues autonomy to the detriment of other ethical principles. A balanced ethical approach would consider the patient's right to treatment, their relationships and interactions with society and not solely the patient's right to liberty and autonomous decision-making

\section{Declaration of interest}

None.

abstract rights rather than empathic contextualised care. Bloch \& Green point out that 'care ethics views utilitarian impartiality and the deontological stress on respect for autonomy as derivatives of perceptions that misrepresent actual relationships between people who are wrestling with day-to-day moral questions. ${ }^{4}$ In contrast, care ethics puts empathy and relationships at the heart of decision-making. Not to help a patient in need is viewed akin to neglect.

Involuntary detention and treatment is one of the most controversial aspects of psychiatric care and has been subject to endless legislation, campaigns, criticism and ethical debate across the globe. The current situation in many countries is that persons who are suspected or known to have a severe mental disorder can be detained and treated against their will under national or regional legislation. Differences exist between the letter of the law, its interpretation, developments of the law through case law, regional custom and practice, and codes of practice where they exist. This can cause varying local practice within similar legislative frameworks. ${ }^{1}$ Ethically, most healthcare legislation tries to find a compromise between the protection of a patient's autonomy and the protection of the patient and others from harm. Autonomy, as part of a rights-based legislative framework, has increasingly dominated our discussions about coercion. However, there are some serious problems with this approach.

(a) Even proponents of autonomy such as the British-Latvian philosopher Isaiah Berlin have emphasised that autonomy can never exist in a vacuum, but always relies on relationships. Thus any autonomous choice will have the potential to interfere with someone else's autonomous choice. Berlin separates two types of liberty - the freedom from something (negative liberty) and the freedom to do something (positive liberty) - to which, he argued, often only the elite of any given society has access. ${ }^{2}$

(b) All serious ethical frameworks consider a number of competing ethical principles, of which autonomy is only one. Most famously, Beauchamp \& Childress postulated beneficence ('do good'), non-maleficence ('do no harm'), autonomy and justice. ${ }^{3}$ These principles were always meant to be taken into account together when solving ethical problems. No preference has been given to any particular one. Indeed, it does not make sense to give a priori preference to any ethical principle. They are not rules, and we all know that there will be times when one is more important than another in any individual situation.

(c) The ethics of care movement has criticised rights-based ethics as predominantly male, and limiting ethics to detached (d) Carson \& Lepping have argued that psychiatry is about helping people with mental illness. By looking at the patient's, carers' and medical narratives and treating them as parallel truths without a priori judgement, the ethic of helping people comes first. This ethic is the foundation of psychiatric practice. 'Although this is a banal truism, it is often overlooked in everyday practice. This is because practising psychiatrists often see their everyday practice as "problem solving". However, solving problems is not the same thing as helping people.. ${ }^{5}$ Problem-solving seems to place the issue firmly within the individual concerned, whereas helping people is about the social context in which the issue occurs. This emphasises the patient's role in the wider context of social relationships, including treating teams.

(e) Patients feel coerced to various degrees regardless of their formal legal status in hospital or the community. In a UK sample the lifetime experience of some form of coercive leverage was reported by $35 \%$ of voluntary psychiatric patients. $^{6}$ Equally, a smaller but sizeable minority of involuntary patients do not feel coerced. Studies have shown that patients who regain insight after mental illness do not, by and large, object to the treating teams having taken benevolent decisions on their behalf. ${ }^{7}$ This shows that the boundaries between voluntary and involuntary are much more complex than an exclusive focus on autonomy might suggest. Autonomy, as asserted by Berlin, always needs to be contextualised and cannot exist in a vacuum without considering relationships.

We suggest that recent developments in psychiatry have favoured the principle of autonomy disproportionately over other ethical principles. By putting most of our ethical eggs in the autonomy basket we have neglected other important principles. This has led to clinical situations in which basic needs of patients such as safety, food and social contacts can get neglected for the higher good of autonomy. In most high-income countries, current, rights-dominated legislation is based on capacity. If patients have capacity to make decisions, their autonomous decision-making trumps all other considerations. However, there 
is reasonable evidence to suggest that clinicians overestimate patients' capacity. ${ }^{8}$ Furthermore, capacity as a concept lacks utility in many acute mental illnesses, with fluctuating capacity and differences of judgement with regard to illness severity and need for treatment. In contrast, it may be a more valid concept in situations of chronic and more cognitive impairment such as dementia or severe intellectual disabilities.

\section{Right to health: Indian context}

In India, the right to health, among others, is protected under the Indian Constitution. Article 21 (Right to life) defines that 'No person shall be deprived of his life or personal liberty except according to procedure established by law. ${ }^{9}$ The right to life is interpreted as more than mere existence and includes the right to live with human dignity and decency. The Indian Mental Health Act 1987 ensures the protection of human rights of the mentally ill. There is an inbuilt assumption that the right to life is more than mere existence, and that the law should protect meaningful existence. The Act incorporates scientific knowledge and social concepts in defining mental illnesses. A modest attempt has been made to bring mental illness onto an equal footing with physical illness, with the intention to reduce stigma. Although the Indian Mental Health Act sanctions coercive procedures during the admission of involuntary patients, patient benefit is its major concern. ${ }^{10}$ In addition, the Act recognises the patient's immediate relationships as part of a meaningful existence. In India, psychiatric care is influenced by a complex web of social, economic, cultural and religious factors. The current Indian legislation does not include any specific provision or definition for involuntary treatment. Thus, coercion by relatives in and outside hospital settings is much more widespread than in Europe, commonly seen as a family obligation and generally more acceptable than it is in high-income countries. ${ }^{10}$ Srinivasan \& Thara reported that Indian families administered covert treatment under the supervision of a psychiatrist in half the cases of nonadherent patients studied. The treatment helped many patients to recover from the illness, enough to voluntarily participate in further treatment, without many negative effects and at a low cost. ${ }^{11}$ Any delay in seeking treatment is mostly attributed to the choice of the first caregivers, who may also make major decisions about treatments for patients who lack capacity. ${ }^{12}$

These contrasting approaches between the Indian and the UK experience highlight a number of key differences regarding individual autonomy. Notwithstanding differences in culture and health provision, the contrast illustrates an important dilemma for practising psychiatrists in the UK and elsewhere: how highly do we regard the patient's autonomy in the context of other considerations? European psychiatry has historically been accused of overriding patient autonomy and practising a form of paternalism. However, the Indian example suggests that decisions that go against the patient's wishes can be developed within a wider set of relationships, especially the patient's relatives. The Indian practice encourages outcome to be looked on as benefitting the patient as well as their immediate relationships. Taking into account the consequences of any treatment decision for the patient's relatives would not necessarily be seen as paternalistic by patients or relatives in India. In contrast, it would be seen as a legitimate aspect of striving for good outcome.

\section{Involuntary treatment: redressing the imbalance}

A psychiatric practice less concerned with the primacy of autonomy would more seriously consider the patient's relationships, their care needs and their long-term social contexts. It would give more importance to the opinions of significant persons in the patient's life, and consider these opinions to form a view of the patient's best interests that is not merely based on theoretical wishes and aims. It would rather look more at what is achievable, with whose help, and in what time frame. The needs of patients with acute symptoms are potentially different to those with chronic problems who are likely to need more support on a long-term basis, making the view of carers more important to take into account (see online supplement for examples of scenarios). Furthermore, the Indian example shows that individual autonomy may not be equally generalisable to cultures where familial interdependence is strong and collective family goals are dominant.

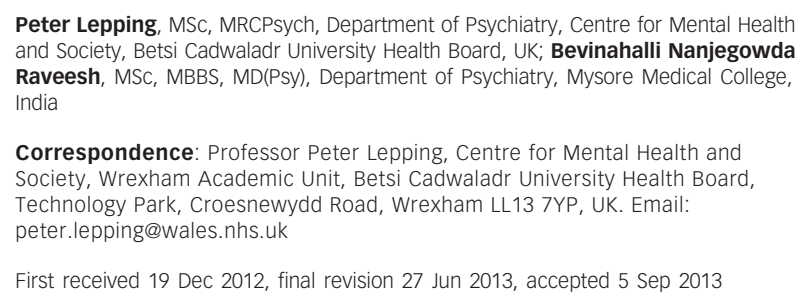

Correspondence: Professor Peter Lepping, Centre for Mental Health and Society, Wrexham Academic Unit, Betsi Cadwaladr University Health Board, Technology Park, Croesnewydd Road, Wrexham LL13 7YP, UK. Email: peter.lepping@wales.nhs.uk

First received 19 Dec 2012, final revision 27 Jun 2013, accepted 5 Sep 2013

\section{Acknowledgement}

We thank Professor Alexander Carson from Glyndŵr University for his help with the revision of this manuscript.

\section{References}

1 Steinert T, Lepping P. Legal provisions and practice in the management of violent patients. A case vignette study in 16 European countries. Eur Psychiatry 2009; 24: 135-41.

2 Berlin I. Four Essays on Liberty. Oxford University Press, 1969 (reprint 2004).

3 Beauchamp T, Childress J. Principles of Biomedical Ethics (5th edn). Oxford University Press, 2001.

4 Bloch S, Green SA. An ethical framework for psychiatry. Br J Psychiatry 2006; 188: 7-12.

5 Carson AM, Lepping P. Ethical psychiatry in an uncertain world: conversations and parallel truths. Philos Ethics Humanit Med 2009; 4: 7.

6 Burns T, Yeeles K, Molodynski A, Nightingale H, Vazquez-Montes M, Sheehan $\mathrm{K}$, et al. Pressures to adhere to treatment ('leverage') in English mental healthcare. Br J Psychiatry 2011; 199: 145-50.

7 Owen GS, David AS, Hayward P, Richardson G, Szmukler G, Hotopf M. Retrospective views of psychiatric in-patients regaining mental capacity. Br J Psychiatry 2009; 195: 403-7.

8 Lepping P. Overestimating patients' capacity. Br J Psychiatry 2011; 199 : $355-6$.

9 Pylee MV. Constitutional Government in India. Chand \& Co, 2004.

10 Shah R, Basu D. Coercion in psychiatric care: global and Indian perspective. Indian J Psychiatry 2010; 52: 203-6.

11 Srinivasan TN, Thara R. At Issue: management of medication noncompliance in schizophrenia by families in India. Schizophr Bull 2002; 28: 531-5.

12 Rajkumar AP, Saravanan B, Jacob KS. Voices of people who have received ECT. Indian J Med Ethics 2007; 4: 157-64. 